\title{
Prevalence of group a streptococcus pharyngeal carriage and clinical manifestations in school children aged 5-15 yrs in Wakiso District, Uganda
}

Irene Nayiga ${ }^{1 *}$, Emmy Okello², Peter Lwabi ${ }^{1,2}$ and Grace Ndeezi ${ }^{1}$

\begin{abstract}
Background: Beta-hemolytic streptococci carrier rates in children living in low-income countries are high ranging from 10 to 50\%. Although most of these children are asymptomatic, they are a reservoir and pose a risk of transmission. The aim of this study was to determine the prevalence of group a streptococcus pharyngeal carriage and clinical manifestations in school going children in Wakiso district, Uganda.

Methods: A cross sectional study targeting children age 5-15 years in primary schools in one sub-county of Wakiso district was carried out. Three hundred and sixty-six children from five primary schools were enrolled and evaluated for group a streptococcus (GAS) carriage. A semi-structured questionnaire was used to collect data that included social demographics, school environment and clinical findings. For every enrolled child a throat swab was taken and cultured for GAS and blood was drawn for anti-streptolysin-O titres. Analysis of data was done using STATA.

Results: The prevalence of GAS carriage was 16\%. The children with GAS positive cultures were mainly females. The factor associated with GAS carriage was the school location, with peri-urban schools more likely to have children with GAS compared to rural schools; AOR 2.48 (95\% Cl: $1.01-6.11), P=0.049$. There was no significant difference between the characteristic of children with GAS positive verses GAS negative throat swab cultures.

Conclusion: There is a high prevalence of GAS pharyngeal carriage among children aged 5-15 years attending primary schools in Wakiso District, Uganda.
\end{abstract}

Keywords: Group a streptococcus, Carriage, Uganda

\section{Background}

Group a beta-hemolytic streptococcus (GAS) has been estimated to account for between 20 to $40 \%$ of all cases of pharyngitis in children [1]. There are 450 million estimated cases of GAS pharyngitis in children each year worldwide [2]. GAS pharyngitis is ubiquitous but is more frequent in low-income countries. Surveys done in healthy school children in the age range of 6 to 10 years found anti-streptolysin-O titers of more than 200 Todd units in 15 to $70 \%$ of the children [3] while other studies

\footnotetext{
* Correspondence: nayigairene@yahoo.com

${ }^{1}$ Department of Paediatrics \& Child Health, College of health sciences,

Makerere University, P.O Box 7072, Kampala, Uganda

Full list of author information is available at the end of the article
}

have reported beta-hemolytic streptococci carrier rates of 10 to $50 \%$ for asymptomatic school children [4].

Group a streptococcus, the most common bacterial cause of pharyngitis, has a peak incidence in children 5-15 years of age [5]. Pharyngitis caused by group a streptococcus has been linked with the aetiopathogenesis of rheumatic fever (RF) and rheumatic heart disease (RHD) [5]. Primary prevention relies on the eradication of group a streptococcal carriage through active sore throat screening and by treatment of pharyngitis with oral antibiotics [6].

The current estimated prevalence of RHD in Kampala, Uganda is 14 per 1000 cases among primary school children and this is a sequeale of group a streptococcus 
pharyngitis [7]. The prevalence of group a streptococcus in school children in Uganda had not been described, despite having several studies done regarding the most adverse sequeale i.e. RF and RHD.

The aims of this study were to determine the prevalence of GAS pharyngeal carriage, describe the clinical characteristics of the children with GAS and determine factors associated with GAS pharyngeal carriage in school going children in Sissa sub-county, Wakiso district, Uganda.

\section{Methods}

This was a cross sectional study targeting children between the ages of 5 to 15 years that were enrolled in primary day schools in Sissa sub-county, Wakiso district. Wakiso district is in the central region of Uganda. It forms a crescent like boundary that almost surrounds Kampala, the capital city of Uganda.

Wakiso is the most populated district in Uganda with $2,007,700$ people. The average household size in this district is 3.9 people. Sissa sub- county lies in the southern part of Wakiso and has a population of 93,238 people.

Children age 5-15 yr. attending primary day schools in Sissa Sub-county, Wakiso district whose parents gave informed consent were enrolled in this study. In addition, assent was attained from pupils aged $>8$ yrs. The children who were on antibiotic treatment during the study period were excluded. Determination of antibiotic use was by self-report at the time of enrollment.

A multi-stage sampling design was employed for this study. Sissa sub-county was randomly selected from the eight sub-counties of Wakiso district with peri-urban and rural populations. Five primary schools Kitende, Bethel, Mpumudde, Jjanyi and Faith Trust were then randomly selected from the list of schools in the subcounty. Using class registers and the assistance of the teachers, eligible children were selected based on the number of children in each class. Every third child starting with the first was selected to participate in the study. The head teachers and school contact persons were informed that any child whose throat swab culture turned positive would be informed and referred to the nearest health center for treatment.

Based on a study by Shaikh $\mathrm{N}$ et al. [1] the GAS carriage prevalence of $12 \%$ was used to calculate the sample size of 325 pupils using the Leslie Kish formula. The sample size for the factors associated was 291 by Hsieh formula. The larger sample size was considered and $10 \%$ was added for non-response.

Asymptomatic GAS pharyngeal carriage was defined as a positive throat culture for GAS without a GAS specific immune response [8]. The immune response considered in this study was antistreptolysin-o antibody titre (ASOT).
Normal values of ASOT are variable and usually depend on the age of the patient, geographical location, epidemiological settings and season of the year [4]. An ASOT level of more than 200 Todd units was taken as generally increased since there was no documentation of the normal values in our setting.

The study examined the school environment such as location [rural, peri-urban], school ownership, number of pupils per class and class floor area. The sociodemographics including age, sex, number of children in the class, number of people at home (household size), type of housing and parent's occupation were recorded for each child. History of fever, sore throat, cough plus documentation of RHD were recorded.

The principal investigator and a medical officer did general exam of the children. This included measuring weight, height, temperature, examination of cervical lymph nodes and the throat. Weights were taken to the nearest $100 \mathrm{~g}$ using a SECA scale. The children were weighed with minimum clothing and standing height was taken using a stadiometer. A body mass index (BMI) was then calculated. A throat swab and blood sample was taken from every child enrolled in the study.

Throat swab collection: In good light, the children opened their mouths as wide as possible. Using a tongue depressor, the investigator looked for inflammation and presence of any exudates or pus in tonsillar area. With a sterile cotton swab, the investigator rubbed on back of the throat and on the tonsils. The swab was then placed in an Amies transport media container, labeled with the study ID number. The sample was placed in a biohazard bag and delivered to the laboratory.

Blood collection for anti-streptolysin O titres (ASOT). The skin around the cubital fossa was cleaned using 70\% isopropyl alcohol. $3 \mathrm{mls}$ of blood was drawn and collected in plain serum (red) top vacutainers.

At the end of the recruitment day, the specimens were transported to a certified clinical laboratory in Kampala for analysis. At the laboratory, the throat swabs were inoculated on blood agar medium. A bacitracin disk was placed in the growth media and incubation was done at $35-37{ }^{\circ} \mathrm{C}$ in an incubator. After $24-48 \mathrm{~h}$, plates were examined for tiny colonies of about $0.5 \mathrm{~mm}$ diameter, with a wider zone of hemolysis. These colonies were subcultured on a new plate of blood agar and susceptibility testing was done.

Susceptibility testing was conducted using the disc diffusion method on Mueller Hinton agar and the antibiotic panel included penicillin, ceftriaxone, vancomycin, erythromycin, azithromycin, tetracycline and chloramphenicol.

A rapid latex agglutination test for the qualitative determination of anti-streptolysin-O (ASO) antibodies in serum was used. A negative result had no agglutination 
of the latex particles suspension and a positive result had an agglutination of the latex particles suspension, indicating an ASO level of more than $200 \mathrm{IU} / \mathrm{ml}$.

In the laboratory, qualified laboratory technicians used standard operating procedures to process the samples and weekly quality control tests were done.

Analysis of data was done using STATA. At the univariate level, means, medians and their measures of dispersion were used to describe continuous variables. Proportions were used to describe categorical variables and the prevalence of GAS carriage. At the bivariate and multivariate analysis, unadjusted and adjusted Odds ratios were used to determine the factors associated with GAS pharyngeal carriage.

\section{Ethical approval}

Permission to carry out the study was sought from School of Medicine Research and Ethics Committee, College of Health Sciences Makerere University, Wakiso district education department, heads of school, parents and children aged 8 years and above. Children with GAS positive cultures received a 10-day course of amoxicillin at government health centers. Amoxicillin is on the essential drug list that the ministry of health Uganda provides to all health facilities.

\section{Results}

Three hundred sixty-six children, aged 5-15 years took part in this study. Enrollment of the study participants took place between 21 July and 29 October 2014 (Fig. 1). The five primary schools selected were Kitende, Bethel, Mpumudde, Jjanyi and Faith trust primary school. Three out of the five are government-aided schools. Kitende, Bethel and Faith trust are peri-urban schools. Bethel primary school has $20 \%$ of its pupils in the day section. The average number of children per square of classroom floor area was 1.3 within the enrolled schools. Table 1 shows the proportions of the pupils enrolled per school in the study.

Of the 366 children, $36.3 \%$ were below 10 years while the rest above 10 years of age. Their mean age was 10 yrs. (SD 2.6). The female children were 217/366 (59.3\%) of the enrolled population. The average household was six people (SD 2.5). Out of the 366 pupils, 241(66\%) were from peri-urban schools. For all the schools the average class size was 63 children (SD 26). Table 2 shows demographic characteristics of the children enrolled.

\section{GAS carriage}

There were $68 / 366(18.5 \%)$ positive throat swab cultures and $35 / 366(9.5 \%)$ positive ASOT tests. Fifty-nine (16.1\%) children had carriage (a positive throat swab culture and a negative ASOT) as shown in Table 3. Of the 59 children

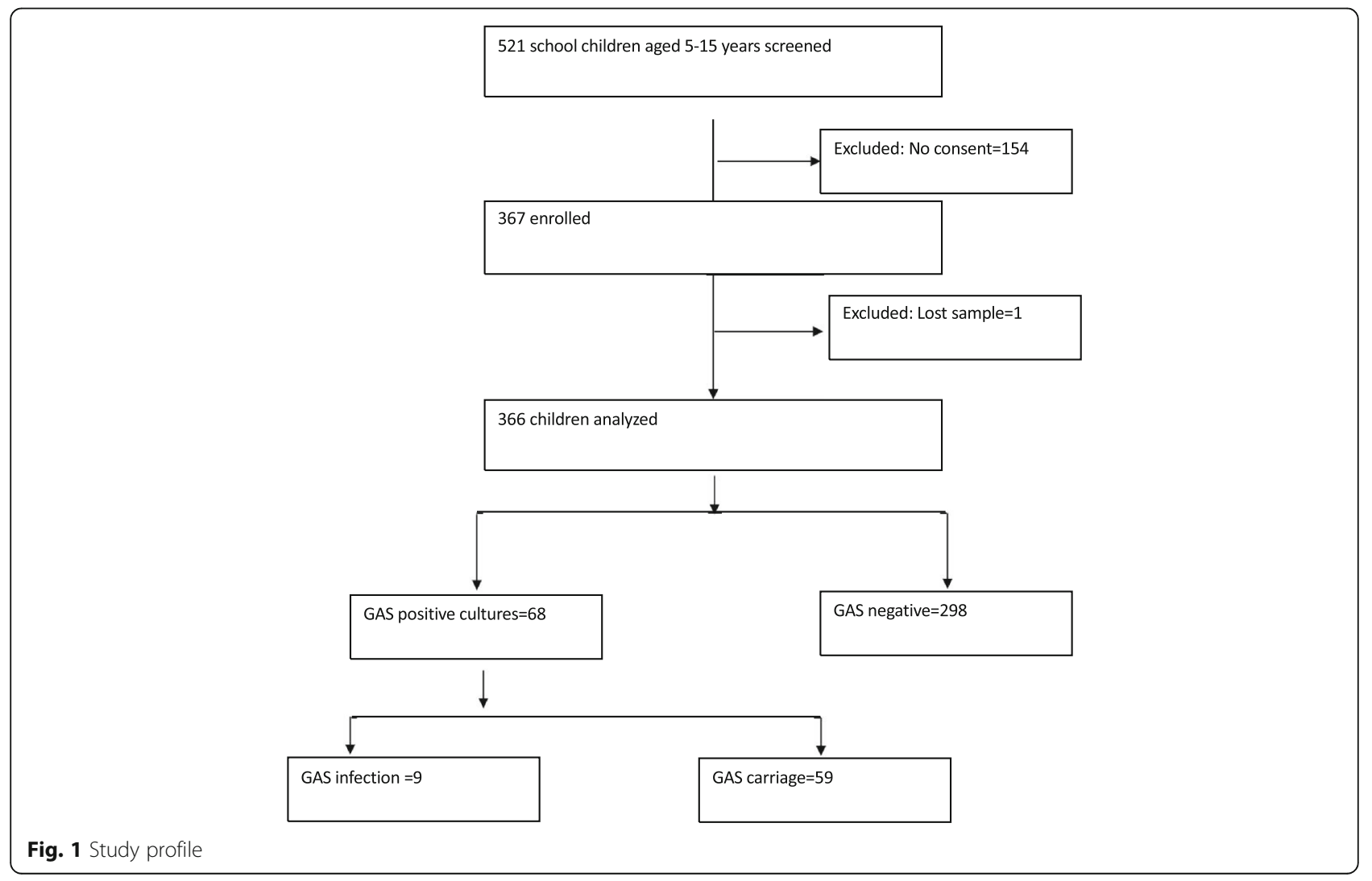


Table 1 School enrolment and classroom space per child in five selected schools in Sissa sub-county, Wakiso

\begin{tabular}{llllll}
\hline School & Total class floor area $\left(\mathrm{m}^{2}\right)$ & Total school enrollment & $\begin{array}{l}\text { Number of children per sq. } \\
\text { meter of floor area }\end{array}$ & $\begin{array}{l}\text { Number enrolled } \\
\text { in the study }\end{array}$ & $\begin{array}{l}\text { Proportion enrolled } \\
\text { in the study (\%) }\end{array}$ \\
\hline Kitende primary & 331.21 & 554 & 1.6 & 119 & 32 \\
Bethel primary & 446.89 & 510 & 1.14 & 58 & 16 \\
Mpumudde primary & 314.87 & 228 & 0.72 & 54 & 15 \\
Jjanyi primary & 376.62 & 270 & 0.72 & 72 & 20 \\
Faith trust primary & 269.99 & 392 & 1.45 & 64 & 17 \\
\hline
\end{tabular}

with carriage, $23.5 \%$ were from Kitende, $29.8 \%$ from Bethel, 9.3\% from Mpumudde, 9.7\% from Jjanyi and 3.1\% from Faith trust primary schools. Nine children, age more than 10 years, had GAS infection (positive throat culture and ASOT).

All the GAS isolates were susceptible to penicillin and vancomycin. The isolates had 80, 76, 61 and 45\% susceptibility to ceftriaxone, azithromycin, erythromycin and tetracycline respectively.

\section{Clinical characteristics of the primary school children with GAS positive throat swab culture enrolled in Sissa sub-county Wakiso}

The children who were culture positive for GAS were 68. The females were $45(66 \%)$ and the mean age was 10.6 yrs. (SD 2.6). Table 4 shows the symptoms and physical findings. Of the 68 children with a GAS positive culture, four children had a fever, sore throat and an abnormality in the throat. One of the four children had positive results for culture and ASOT. There was no child with a documented history of RHD.

\section{Factors associated with GAS carriage}

At bivariate analysis, the children from peri-urban compared to rural schools were 2 times more likely to have carriage (Table 5). Children from classes of more than 0.8 children per square meter were also 2 times more likely to have carriage.

All factors, whose $p$-value were less than 0.2 , were subjected to logistic regression. Factors that were known to influence the outcome were included in the multivariate analysis even if their $p$ value was more than 0.2 . The only factor associated with GAS carriage was school location. The peri-urban schools were associated with GAS carriage; $p$-value was 0.049 as shown in Table 6.

Table 2 Social demographic characteristics of the children enrolled in Sissa sub-county, Wakiso

\begin{tabular}{|c|c|c|c|c|c|c|}
\hline \multirow[t]{3}{*}{ Variable } & \multicolumn{5}{|l|}{ School } & \multirow[t]{2}{*}{ Total } \\
\hline & Kitende & Bethel & Mpumudde & Jjanyi & Faith Trust & \\
\hline & $N(\%)$ & $N(\%)$ & $N(\%)$ & $N(\%)$ & $N(\%)$ & $N(\%)$ \\
\hline \multicolumn{7}{|l|}{ Sex: } \\
\hline Male & $58(48.3)$ & $14(24.6)$ & $20(37.0)$ & $31(43.1)$ & $26(40.6)$ & $149(40.7)$ \\
\hline Female & $61(51.7)$ & $43(75.4)$ & $34(63.0)$ & $41(56.1)$ & $38(59.4)$ & $217(59.3)$ \\
\hline \multicolumn{7}{|l|}{ House Type $^{a}$} \\
\hline Single & $72(60.5)$ & $35(61.4)$ & $44(81.5)$ & $44(61.1)$ & 49 (76.6) & $244(68)$ \\
\hline Semi detached & $44(37.0)$ & $18(31.6)$ & $10(18.5)$ & $28(38.9)$ & $15(23.4)$ & $115(32)$ \\
\hline \multicolumn{7}{|l|}{ School Location: } \\
\hline Rural & 0 & 0 & 54 & 72 & 0 & $125(34)$ \\
\hline Peri-urban & 119 & 57 & 0 & 0 & 64 & $241(66)$ \\
\hline \multicolumn{7}{|c|}{ School Type Child Attends: } \\
\hline Private & 0 & 57 & 0 & 0 & 64 & 121(33) \\
\hline Government & 119 & 0 & 54 & 72 & 0 & $245(67)$ \\
\hline \multicolumn{7}{|l|}{ Class child Attends: } \\
\hline Lower & 45 (37.8) & $21(36.8)$ & $27(50.0)$ & $45(62.5)$ & $30(46.9)$ & 168(45.9) \\
\hline Middle & $49(41.2)$ & 9 (15.8) & $15(27.8)$ & 22 (30.6) & 26 (40.6) & 121(33.1) \\
\hline Upper & $25(21.0)$ & $27(47.4)$ & $12(22.2)$ & $5(6.9)$ & $8(12.5)$ & $77(21)$ \\
\hline
\end{tabular}

${ }^{\mathrm{a}} 7$ children had this information missing 
Table 3 A comparison of the throat swab culture and ASOT tests for children attending Primary Schools in Sissa sub-county, Wakiso

\begin{tabular}{lll}
\hline & $\begin{array}{l}\text { ASOT } \\
\text { Positive }(n=35) \\
N(\%)\end{array}$ & $\begin{array}{l}\text { ASOT } \\
\text { Negative }(n=331) \\
N(\%)\end{array}$ \\
\hline $\begin{array}{l}\text { Throat swab culture: } \\
\text { Positive }(n=68)\end{array}$ & $9(2.5)$ & $59(16.1)$ \\
Negative $(n=298)$ & $26(7.1)$ & $272(74.3)$ \\
\hline
\end{tabular}

\section{Discussion}

This study describes the prevalence of GAS and factors associated with GAS pharyngeal carriage among children attending primary schools in one of the most populated districts of Uganda, Wakiso district.

The prevalence of GAS pharyngeal carriage was $16 \%$. In the peri-urban schools, 1 in 5 children had GAS carriage. The prevalence in this study is slightly higher than the $12 \%$ pooled prevalence in a meta-analysis by Shaikh et al. [1] and that of a study done in the Ethiopian cities of Addis Ababa, Gondar and Dire Daua that was at 9.7\% [9]. The prevalence is still higher than that found by
Engel et al. of 3\% among healthy school children in the lower socio class communities of Cape Town [10]. The high crowding index in Sissa primary schools, especially in the urban schools, is the probable cause of the high prevalence.

During the assessment for GAS carriage, 2.5\% of the children had GAS infection. This proportion may be an under-estimate since most children with GAS infection are likely to be symptomatic and likely to stay at home during illness. However, a small number of children with active infection still attend school and probably do not get medical attention. In a clinic-based study in South Africa, the prevalence was $21.6 \%$ among children who presented with a history of sore throat [11]. In the current study, the children were relatively well and this explains the low prevalence of active infection as opposed to studies that have assessed symptomatic/sick children.

A few children had a positive ASOT test but a negative throat swab culture. This reflects either antecedent GAS infection or may be infection secondary to group $C$ or $\mathrm{G}$ beta -hemolytic infection [4, 12]. ASOT may

Table 4 Characteristics of the primary school children with GAS positive versus GAS negative throat swab culture in Sissa subcounty, Wakiso

\begin{tabular}{|c|c|c|c|c|}
\hline Characteristic & GAS positive $N(\%)$ & GAS negative $N(\%)$ & OR(95\% C.I) & $P$ value \\
\hline \multicolumn{5}{|l|}{ Sex } \\
\hline Male & $23(33.8)$ & $126(42.3)$ & $1.43(0.83-2.49)$ & 0.200 \\
\hline Female & $45(66.2)$ & $172(57.7)$ & & \\
\hline \multicolumn{5}{|l|}{ Age } \\
\hline$<10 y$ & $32(47.1)$ & $162(54.4)$ & $1.34(0.79-2.27)$ & 0.277 \\
\hline$>10 \mathrm{yr}$ & $36(52.9)$ & $136(45.6)$ & & \\
\hline \multicolumn{5}{|l|}{ History of fever } \\
\hline Yes & $4(5.9)$ & $15(5)$ & $1.18(0.38-3.67)$ & 0.776 \\
\hline No & $64(94.1)$ & 283(95) & & \\
\hline \multicolumn{5}{|l|}{ Cough } \\
\hline Yes & $31(45.6)$ & $110(36.9)$ & $1.43(0.84-2.44)$ & 0.185 \\
\hline No & $37(54.4)$ & 188(63.1) & & \\
\hline \multicolumn{5}{|l|}{ Sore throat } \\
\hline Yes & $8(11.8)$ & $61(20.5)$ & $0.52(0.24-1.14)$ & 0.098 \\
\hline No & $60(88.2)$ & $237(79.5)$ & & \\
\hline \multicolumn{5}{|c|}{ Palatal petechae } \\
\hline Yes & $2(2.9)$ & $23(7.7)$ & $0.36(0.08-1.58)$ & 0.159 \\
\hline No & $66(97.1)$ & 275(92.3) & & \\
\hline \multicolumn{5}{|c|}{ Tonsillar enlargement } \\
\hline Yes & $6(8.8)$ & $50(16.8)$ & $0.48(0.20-1.17)$ & 0.100 \\
\hline No & $62(91.2)$ & $248(83.2)$ & & \\
\hline \multicolumn{5}{|c|}{ Cervical adenopathy } \\
\hline Yes & $9(13.2)$ & $57(19.1)$ & $0.64(0.30-1.17)$ & 0.254 \\
\hline No & $59(86.8)$ & $241(80.9)$ & & \\
\hline
\end{tabular}


Table 5 Bivariate analysis of the factors associated with GAS carriage among primary school children in Sissa sub-county, Wakiso

\begin{tabular}{|c|c|c|c|c|c|}
\hline \multirow[t]{2}{*}{ Variables } & \multicolumn{2}{|l|}{ GAS carriage } & \multirow[b]{2}{*}{ unadjusted OR } & \multirow[b]{2}{*}{$95 \%$ C.I } & \multirow[b]{2}{*}{$P$-value } \\
\hline & No & Yes & & & \\
\hline Age greater $<10$ years & $163(84.0 \%)$ & $31(16.0 \%)$ & & & \\
\hline Age greater $>10 \mathrm{yrs}$ & $114(83.7 \%)$ & $28(16.3 \%)$ & 1.029 & $0.589-1.797$ & 0.921 \\
\hline male & $128(85.9 \%)$ & $21(14.1 \%)$ & & & \\
\hline Female & $179(82.5 \%)$ & $38(17.5 \%)$ & 1.304 & $0.731-2.327$ & 0.369 \\
\hline Rural school & $113(90.4 \%)$ & $12(9.6 \%)$ & & & \\
\hline Peri urban school & $194(80.5 \%)$ & 47 (19.5\%) & 2.270 & $1.156-4.457$ & 0.017 \\
\hline Government school & $205(83.7 \%)$ & $40(16.3 \%)$ & & & \\
\hline Private school & $102(84.3 \%)$ & $19(15.7 \%)$ & 0.945 & $0.521-1.714$ & 0.853 \\
\hline Household with $>4$ people & $222(82.8 \%)$ & $46(17.2 \%)$ & & & \\
\hline Household with 4 or less people & $84(87.5 \%)$ & $12(12.5 \%)$ & 0.693 & $0.35-1.371$ & 0.292 \\
\hline Class with $>3$ windows & $154(85.6 \%)$ & $26(14.4 \%)$ & & & \\
\hline class with 3 or less windows & $153(82.3 \%)$ & $33(17.7 \%)$ & 1.269 & $0.725-2.223$ & 0.404 \\
\hline Semi-detached house & $98(86.0 \%)$ & $16(14.0 \%)$ & & & \\
\hline Singles house & $204(83.6 \%)$ & $40(16.4 \%)$ & 1.213 & $0.648-2.272$ & 0.546 \\
\hline \multicolumn{6}{|l|}{ Fever: } \\
\hline no & $290(83.6 \%)$ & $57(16.4 \%)$ & & & \\
\hline yes & 17 (89.5\%) & $2(10.5 \%)$ & 0.601 & $0.135-2.671$ & 0.503 \\
\hline \multicolumn{6}{|l|}{ Sore throat: } \\
\hline no & $245(82.5 \%)$ & $52(17.5 \%)$ & & & \\
\hline yes & 62 (89.9\%) & $7(10.1 \%)$ & 0.534 & $0.231-1.233$ & 0.142 \\
\hline \multicolumn{6}{|l|}{ Cough: } \\
\hline no & $192(85.3 \%)$ & $33(14.7 \%)$ & & & \\
\hline yes & $115(81.6 \%)$ & $26(18.4 \%)$ & 1.322 & $0.753-2.323$ & 0.331 \\
\hline \multicolumn{6}{|l|}{ Palatal petechiea } \\
\hline no & $284(83.3 \%)$ & $57(16.7 \%)$ & & & \\
\hline yes & $23(92.0 \%)$ & $2(8.0 \%)$ & 0.435 & $0.1-1.896$ & 0.268 \\
\hline \multicolumn{6}{|l|}{ Tonsillar enlargement: } \\
\hline no & $256(82.6 \%)$ & $54(17.4 \%)$ & & & \\
\hline Yes & $51(91.1 \%)$ & $5(8.9 \%)$ & 0.467 & $0.178-1.224$ & 0.121 \\
\hline \multicolumn{6}{|l|}{ Cervical nodes: } \\
\hline no & $249(83.0 \%)$ & $51(17.0 \%)$ & & & \\
\hline yes & $58(87.9 \%)$ & $8(12.1 \%)$ & 0.676 & $0.304-1.502$ & 0.337 \\
\hline \multicolumn{6}{|l|}{ Parent's employment: } \\
\hline informal & $256(83.9 \%)$ & 49 (16.1\%) & & & \\
\hline Formal & $46(86.8 \%)$ & $7(13.2 \%)$ & 0.795 & $0.339-1.864$ & 0.598 \\
\hline \multicolumn{6}{|l|}{ Congestion: } \\
\hline$<0.8$ children/m2 & $51(91.1 \%)$ & $5(8.9 \%)$ & & & \\
\hline$>0.8$ children/m2 & $256(82.6 \%)$ & $54(17.4 \%)$ & 2.152 & $0.820-5.643$ & 0.119 \\
\hline \multicolumn{6}{|l|}{ BMl } \\
\hline Underweight & $9(81.8 \%)$ & $2(18.2 \%)$ & 1.000 & & \\
\hline Normal & $294(83.8 \%)$ & $57(16.2)$ & 0.872 & $0.184-4.144$ & 0.864 \\
\hline Over weight & 4 (100.0\%) & $0(0.0 \%)$ & 1.000 & & \\
\hline
\end{tabular}


Table 6 Multivariate analysis for the factors associated with GAS carriage among primary school children in Sissa sub-county Wakiso

\begin{tabular}{|c|c|c|c|c|c|c|}
\hline & \multicolumn{3}{|c|}{ Unadjusted } & \multicolumn{3}{|c|}{ Adjusted } \\
\hline & $\mathrm{OR}$ & $95 \% \mathrm{Cl}$ & $p$-value & $\mathrm{OR}$ & $95 \% \mathrm{Cl}$ & $P$-value \\
\hline Peri-urban & 2.270 & $1.156-4.457$ & 0.017 & 2.477 & $1.005-6.107$ & 0.049 \\
\hline Sore throat & 0.534 & $0.231-1.233$ & 0.142 & 0.447 & $0.179-1.116$ & 0.085 \\
\hline Tonsillar enlargement & 0.467 & $0.178-1.224$ & 0.121 & 0.650 & $0.235-1.796$ & 0.406 \\
\hline Parent's employment & 0.795 & $0.339-1.864$ & 0.598 & 0.629 & $0.257-1.537$ & 0.309 \\
\hline Female & 1.304 & $0.731-2.327$ & 0.369 & 1.269 & $0.69-2.332$ & 0.443 \\
\hline Household with less than 4 & 0.693 & $0.35-1.371$ & 0.292 & 0.628 & $0.301-1.309$ & 0.215 \\
\hline Palatal petechiea & 0.467 & $0.178-1.224$ & 0.121 & 0.593 & $0.129-2.725$ & 0.502 \\
\hline Congestion >0.8children /m2 & 2.152 & $0.820-5.643$ & 0.119 & 1.013 & $0.297-3.461$ & 0.983 \\
\hline
\end{tabular}

remain positive for several months following an initial infection [12].

Most of the children with GAS positive culture had at least a symptom or a sign. The children with GAS positive infection usually present with sudden onset of sore throat, fever, patchy tonsillopharyngeal exudates, palatal petechiae and anterior cervical adenitis (tender nodes) [13].

Two thirds of the children with symptoms were females and for this study, there were more females evaluated than males. Therefore, the high percentage of females with GAS could probably be attributed to the slightly high percentage of females in the overall sample size. In a South African study, they found equal distribution of GAS positive findings between the sexes [10].

Quite a number of children with a positive throat swab culture had a cough. Cough is not a common symptom of GAS carriage nor infection. Cough, as a symptom has been associated with viral causes in patients who also have a sore throat [13]. Since we were assessing for carriage this reflects probably carriage with inter-current viral infections in the study population.

There were quite a number of children with signs and symptoms but a negative culture. This could have been due to other non-streptococcal causes (viruses) or previous antibiotic use. Viruses are the commonest cause of pharyngitis [5]. Other bacterial causes of pharyngitis include group C and G beta-hemolytic streptococci [4].

There were a few children with sore throat, fever and tonsillar enlargement found with GAS positive culture. The majority of children did not have clinical features because children with symptoms were more likely to have stayed at home at the time of the examination. The other reason was that we were assessing relatively well children.

Asymptomatic carriage of GAS is common among contacts of patients with GAS pharyngitis [13]. Almost a third of individuals with pharyngitis that warrant testing and treatment have been in contact with a GAS carrier. In our study, all children with a positive throat culture for GAS received treatment. Most literature though does not support treatment of GAS carriers because of their low risk of transmission.

Several factors have been associated with GAS carriage among children. The factor that was associated with GAS carriage in this study was school location. Children in peri-urban schools were 2 times more likely to have GAS carriage. This finding is similar to that found in Fiji schools where GAS carriage was associated with peri-urban schools [14]. The study conducted in Fiji, had a similar population and setting as in our study.

The current ministry of education Uganda recommendation is 0.8 pupils per square meter i.e. not less than $51.04 \mathrm{~m}^{2}$ of floor area for pupils not more than 40 per class (Uganda licensing and registration guidelines of private schools in Uganda). The crowding index in the classes was 1.3 children per classroom floor area with the peri-urban schools having a higher index compared with the rural schools.

The average household in this study was six people though we were not able to assess the floor area in the homes of the participants. Hence, we were not able to determine whether this influenced the high prevalence.

In Chennai, India among slum children aged 515 yrs., factors that were significantly associated with GAS isolation were the father's occupation, number of windows on the family house and age [15]. Our study, did not find age nor guardian's occupation to be associated with GAS carriage.

In South Africa, among the lower socioeconomic populations, carriage was neither associated with gender nor age [10]. These populations are similar to ours though the economic status may be different despite both being of low social class.

This study had several limitations. Winter and rainy seasons have high peaks of GAS colonization [16]. The study took place during a dry season and the effect of this on GAS carriage cannot be ascertained.

Sick children who did not attend school missed out and the likely probability that they did harbor the 
organism might have affected our findings. This may also explain why we did not find any children with history of RHD.

We were not able to determine the household floor space for the participants to determine whether this would have influenced our outcome. The participants spend their daytime both at home and school.

Anaerobic cultures may increase the proportion of positive culture results. Because of limited funds, aerobic cultures were used and these yield $90-95 \%$ positive results [13].

Recent antibiotic use was not included due to challenges of documentation and off the counter self-prescriptions in our setting. This may have affected our results.

\section{Conclusion}

In this study, the prevalence of GAS was high with the peri-urban schools having higher rates of carriage. In Sissa sub-county Wakiso, the high crowding index in peri- urban schools may have increased the risk of GAS colonization among pupils.

\section{Additional file}

Additional file 1: Group a streptococcus dataset Wakiso, Uganda (XLS 133 kb)

\section{Abbreviations}

GAS: Group a beta-hemolytic streptococcus; RF: Rheumatic fever; RHD: Rheumatic heart disease; ASOT: Anti-streptolysin O titres; BMI: Body mass index

\section{Acknowledgements}

Paul Mbaka participated in data analysis.

Assimwe Fortunate and Aidah Nakate participated in the data collection.

Department of Paediatrics, Makerere University College of health sciences.

\section{Funding}

Research reported in this publication was supported by the Fogarty International Center of the National Institutes of Health under award number R24TW008861. The content is solely the responsibility of the authors and does not necessarily represent the official views of the National Institutes of Health.

\section{Availability of data and materials}

The datasets analyzed during the study are attached as Additional file 1.

\section{Authors' contributions}

$\mathrm{NI}$ was involved in the concept, design, data acquisition, analysis and writing of the manuscript. OE was involved in the concept, design and revision of the manuscript. LP was involved in the concept, design, data analysis and revision of the manuscript. NG was involved in the concept, design, data analysis and revision of the manuscript. All authors read and approved the final manuscript.

\section{Competing interests}

The authors declare that they have no competing interests.

\section{Consent for publication}

Not applicable.

\section{Ethics approval and consent to participate}

Permission to carry out the study was sought from School of Medicine Research and Ethics Committee, College of Health Sciences Makerere
University, Wakiso district education department and the head teachers. Parents provided written informed consent to participate in the study. Assent was also obtained from children aged 8 years and above.

\section{Publisher's Note}

Springer Nature remains neutral with regard to jurisdictional claims in published maps and institutional affiliations.

\section{Author details}

${ }^{1}$ Department of Paediatrics \& Child Health, College of health sciences, Makerere University, P.O Box 7072, Kampala, Uganda. ${ }^{2}$ Uganda Heart Institute, Mulago Hospital Complex, PO Box 7051, Kampala, Uganda.

Received: 28 November 2016 Accepted: 28 March 2017

Published online: 05 April 2017

\section{References}

1. Shaikh N, Leonard E, Martin JM. Prevalence of streptococcal pharyngitis and streptococcal carriage in children: a meta-analysis. Pediatrics. 2010;126(3): e557-e64.

2. Carapetis JR, Steer AC, Mulholland EK, Weber M. The global burden of group a streptococcal diseases. Lancet Infect Dis. 2005;5(11):685-94.

3. Taranta A, Markowitz M. Rheumatic fever: Kluwer academic publishers; 1989

4. Organization WH. Rheumatic fever and rheumatic heart disease: report of a WHO expert consultation, Geneva, 20 October-1 November 2001: World Health Organization; 2004

5. Bisno AL. Acute pharyngitis: etiology and diagnosis. Pediatrics. 1996; 97(6):949-54.

6. Marijon E, Mirabel M, Celermajer DS, Jouven X. Rheumatic heart disease. Lancet. 2012;379(9819):953-64.

7. Okello E, Kakande B, Sebatta E, Kayima J, Kuteesa M, Mutatina B, et al. Socioeconomic and environmental risk factors among rheumatic heart disease patients in Uganda. PLoS One. 2012;7(8):e43917.

8. Kliegman R. Nelson textbook of pediatrics: Saunders Elsevier Philadelphia; 2007.

9. Abdissa A, Asrat D, Kronvall G, Shitu B, Achiko D, Zeidan M, et al. Throat carriage rate and antimicrobial susceptibility pattern of group a streptococci (GAS) in healthy Ethiopian school children. Ethiop Med J. 2011:49(2):125-30.

10. Engel ME. A study of determinants and prevalence of rheumatic heart disease in cape Town: University of Cape Town; 2012.

11. Engel ME, Muhamed B, Whitelaw AC, Musvosvi M, Mayosi BM, Dale JB. Group a streptococcal emm type prevalence among symptomatic children in cape Town and potential vaccine coverage. Pediatr Infect Dis J. 2014;33(2):208.

12. Johnson DR, Kurlan R, Leckman J, Kaplan EL. The human immune response to streptococcal extracellular antigens: clinical, diagnostic, and potential pathogenetic implications. Clin Infect Dis. 2010;50(4):481-90.

13. Shulman ST, Bisno AL, Clegg HW, Gerber MA, Kaplan EL, Lee G, et al. Clinical practice guideline for the diagnosis and management of group $A$ streptococcal pharyngitis: 2012 update by the Infectious Diseases Society of America. Clinical infectious diseases. 2012:cis629.

14. Steer AC, Jenney AW, Kado J, Good MF, Batzloff M, Magor G, et al. Prospective surveillance of streptococcal sore throat in a tropical country. Pediatr Infect Dis J. 2009;28(6):477-82.

15. Kalpana S, Sundar JS, Parameshwari S, Kuganantham P, Selvam JM, Valarmathi MS, et al. Isolation and Identification of Group A Streptococcal Infection Among Slum Children in the Age Group of 5-15 Years in ChennaiOne Year Prospective Study. Age. 2012;5(10yrs):313.

16. Nandi S, Kumar R, Ray P, Vohra H, Ganguly NK. Group a streptococcal sore throat in a periurban population of northern India: a one-year prospective study. Bull World Health Organ. 2001;79(6):528-33. 